\title{
Novel RGS materials with high fill factors and no material-induced shunts with record solar cell efficiencies exceeding $16 \%$
}

\author{
Y.P. Botchak Mouafi ${ }^{\text {a,* }}$, A.S. Zuschlag ${ }^{\text {a, }}$ P.-Y. Pichon ${ }^{\text {b }}$, J.M. Fritz ${ }^{\text {a }}$, A. Schönecker ${ }^{\text {b }}$, G. Hahn ${ }^{\text {a }}$ \\ a University of Konstanz, Department of Physics, 78457 Konstanz, Germany \\ ${ }^{\mathrm{b}}$ RGS Development B.V., Bijlestaal 54a, 1721 PW Broek op Langedijk, The Netherlands
}

\section{A R T I C L E I N F O}

Keywords:

Ribbon growth on substrate

RGS

Kerfless

Shunt

Current collecting structures

PERC solar cell

\begin{abstract}
A B S T R A C T
Kerf losses due to ingot and wafer sawing can be avoided by solidifying the silicon wafers directly from the melt by the Ribbon Growth on Substrate (RGS) process, thus significantly reducing the wafer cost. However, up to now solar cells made from standard RGS material suffered from shunting problems due to current collecting structures. This resulted in lower fill factor values and hence in lower efficiencies compared to solar cells made from block cast multicrystalline silicon (mc Si) materials. In this con tribution two novel RGS materials are presented and investigated. Solar cells processed from these new materials have fill factor values above 78\%, comparable to those of mc Si. The increased fill factor values can be explained by the absence of current collecting structures as concluded from a comparative analysis of spatially resolved Light Beam Induced Current (LBIC) measurements and Electroluminescence (EL) images, and from infrared transmission microscopy investigations. Additionally the improved material quality resulted in open circuit voltage $V_{\mathrm{OC}}$ values up to $608 \mathrm{mV}$. This enhanced material quality, in combination with increased fill factor values, resulted in record efficiencies above $16 \%$ (certified by Fraunhofer ISE CalLab). This represents a significant improvement compared to the former efficiency record of $14.4 \%$ for standard RGS material.
\end{abstract}

\section{Introduction}

Nowadays the wafers' cost represents about $40 \%$ of the crys talline silicon (c Si) PV module production cost [1 3]. Therefore, besides the reduction of the solar cell processing cost, the reduc tion of the wafer cost is an important factor to reduce the price per watt peak at module level. Currently the silicon PV industry widely uses wafers sawn from ingots after crystallization. Through the wafer sawing process $4555 \%$ of the silicon is lost (this material loss is called kerf loss) [2]. Kerf losses can be suppressed by crys tallizing silicon wafers directly from the silicon melt using ribbon growth techniques such as Edge defined Film fed Growth (EFG), String Ribbon (SR), Ribbon on Sacrificial Template (RST), Direct Wafer (from 1366 Technologies [4]) or Ribbon Growth on Sub strate (RGS) [5]. In the RGS process, introduced in 1990 by Lange and Schwirtlich [6], the crystal growth velocity and the material production speed are decoupled, allowing a higher production speed and lower energy consumption compared to other ribbon techniques $[7,8]$. Indeed typical material production speeds are $\sim 650 \mathrm{~cm} / \mathrm{min}$ for RGS, $510 \mathrm{~cm} / \mathrm{min}$ for RST, and $12 \mathrm{~cm} / \mathrm{min}$ for

\footnotetext{
* Corresponding author. Tel.: +49 7531 882082; fax: +49 7531883895 .

E-mail address: yves.patrick.botchak@uni-konstanz.de (Y.P. Botchak Mouafi).
}

EFG and SR [9]. However, for a fair comparison of the cost effectiveness of all these ribbon techniques, also the thickness of the obtained wafer has to be taken into account regarding the material saving (here, ribbon technologies such as RST are espe cially distinguished due to the possibility to produce very thin wafer with thicknesses down to $60 \mu \mathrm{m}[9,10])$. Hence the RGS process provides cost effective alternative wafer materials for solar cell fabrication. However, compared to ingot grown mc Si materials, the RGS material suffers from lower minority carrier lifetimes due to a higher density of structural defects (grain size $\sim 0.2 \mathrm{~mm}$, significantly higher dislocation density), and from relatively higher impurity concentrations (in particular $\left[C_{s}\right]>10^{18} \mathrm{~cm}^{3}$, i.e. above the solubility limit, $\left[\mathrm{O}_{\mathrm{i}}\right]>10^{17} \mathrm{~cm}^{3}$ ). Since these material properties are closely related to the solidifi cation conditions, we shall now shortly review some important aspects of silicon growth in the RGS process.

A scheme of the process is shown in Fig. 1. The whole process runs under argon. The liquid silicon bath is contained into a casting frame continuously replenished by silicon melted in a crucible placed above (not shown in Fig. 1). Substrates of the same dimensions of the wafer are transported at a constant velocity under the silicon bath. The substrates are a few hundred degrees colder than the silicon melting point and therefore provide the driving force for nucleation at the entrance of the casting frame 


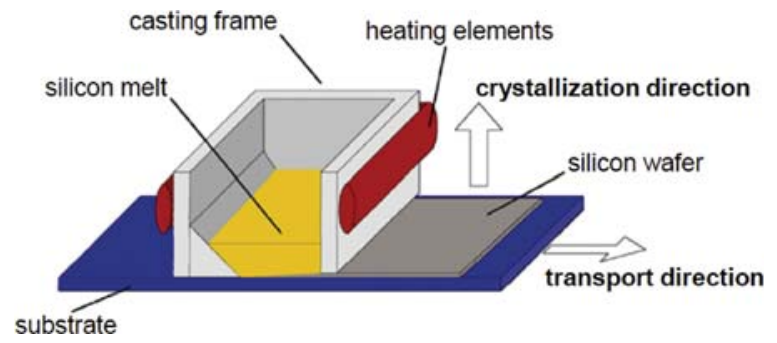

Fig. 1. RGS wafer production principle [11].

and for removal of the crystallization heat during the residence time under the silicon bath. The difference of thermal expansion coefficients between the wafer and the substrates results in stresses at the substrate wafer interface during cooling and in the spontaneous detachment of the solidified material. The wafer can then be picked out of the process chamber and the substrate can then be reused.

An imperfect thermal mechanical contact is realized between the substrate and the silicon that controls the heat transfer between the growing wafer on the one hand, and the adhesion force between the solidified wafer and the substrate that affects the detaching behavior on the other hand. This imperfect contact consists in discrete contact spots that make a small fraction (typically in the range of $0.15 \%$ ) of the total substrate surface area. The position of the contact spots can be controlled by suitable substrate topologies.

In the RGS process the liquid silicon is processed in contact with graphite parts (casting frame, melting crucible, etc.) and therefore is saturated with carbon to the liquid solubility limit. This results in the growth of precipitated SiC filaments in the RGS material. The mechanisms of carbon segregation, SiC filament precipitation and growth at grain boundaries during wafer soli dification were elucidated by Pichon et al. in previous research [12]. In a separate study on RGS material Hess et al. showed that the $\mathrm{SiC}$ precipitates were electrically active and formed current collecting structures responsible for fill factor values below $72 \%$, i.e. the conductive SiC filaments caused shunting [13]. Similar conclusions were reached for block cast mc Si [14 16]. As a result, up to now these current collecting structures prevented from reaching competitive cell efficiencies [7,13]: the highest efficiency obtained on RGS material was $14.4 \%$ [17]. Thus, so far competitive solar cell efficiencies with RGS material were conditioned to avoiding the formation of $\mathrm{SiC}$ filament precipitates and the cor responding current collecting structures. This work focuses on the characterization of two new promising RGS materials. In parti cular, we show that current collecting structures are absent on these materials, allowing to reach significantly higher efficiencies.

The paper is organized as follows. In the 'experiment' section we give a short description of the production and properties of the new RGS materials, and we present the solar cell process used to evaluate their properties. In the 'results and discussion' section we first give an overview of the results by discussing the IV char acteristics of solar cells from each material. Finally, we show a detailed analysis performed on one representative solar cell of each material group to shed some light on the reasons for the improvement in solar cell efficiency for the new RGS materials.

\section{Experiment}

In the first experimental section we will describe the growth characteristics of the new materials and the important related material properties. To determine the potential of the novel RGS materials at cell level, a lab type Passivated Emitter and Rear Cell
(PERC) process was applied on the different material groups: the new RGS materials, a block cast mc Si reference material and standard RGS material. This process will be described in the sec ond section.

\subsection{Production and properties of the investigated RGS materials}

The two novel RGS materials characterized in the present work will be referred to as RGS_A and RGS_B. For comparison block cast mc Si (p type, $1.4 \Omega \mathrm{cm}$ ) and standard RGS material (RGS_Std) were also processed into solar cells. All RGS materials were pro duced in a RGS lab type production tool. RGS_B and RGS_Std wafers were produced in the same RGS casting experiment, whereas RGS_A material originates from another casting run. All RGS materials were crystallized on topologically patterned sub strates: the pattern consisted of a 2D array of pillars arranged in a square lattice, resulting in the same arrangement of the contact spots between the wafer and the substrate. Unlike the standard RGS material, the novel RGS materials were cast on monocrystal line silicon substrates. Separate fundamental research on wetting between liquid silicon and a silicon substrate showed that under certain conditions the same scarcity of contact spots could be achieved on monocrystalline silicon substrate as with standard substrates. Briefly, this seemingly unexpected behavior originates from the fact that under RGS non isothermal wetting conditions, solidification 'at' the spreading contact line prevents steady state wetting to be reached [18]. Thus, thermodynamically 'good wet ting material' for silicon (like $\mathrm{Si}_{3} \mathrm{~N}_{4}, \mathrm{SiC}$ or $\mathrm{Si}$ as a substrate) can behave like non wetting ones, and in particular when the substrate is patterned, composite wetting can be achieved (so called 'lotus leaf effect').

For the new RGS materials, casting conditions were found such as both a small amount of thermal mechanical contact and the transfer of the crystalline orientation from the substrate to the growing material by growth from the substrate were achieved. This lead to new RGS materials with a dominant crystal orienta tion: (111) for RGS_A and (100) for RGS_B [18], as shown by the EBSD measurements in Fig. 2. Fig. 2 also shows that as a result of crystal orientation control, large angle grain boundaries are very rare in the new materials, while they are dominant for the stan dard material.

Unlike the standard material, in the case of silicon substrates there is no difference in thermal expansion coefficient between the wafer and the substrate. Therefore, it was needed to apply, $a$ posteriori, a small mechanical stress to detach the solidified layer and break the contact spots.

Although the crystallographic orientations are not relevant for the solar cell process used here because of the isotropic character of the applied plasma texture, we decided to define the samples as described above to keep consistency with the sample identifica tions and future studies. Note that an alkaline surface texture can be applied on the (100) oriented material. In contrast, the standard RGS material has a fully random grain orientation [11]. Both the new and the standard RGS materials are boron doped (p type). The resistivity of the RGS materials was determined by Quasi Steady State Photo Conductance decay (QSSPC), where only the elec trically active boron atoms contribute to the measured resistivity [19]. The novel RGS materials show an average resistivity value of $\sim 0.7 \Omega \mathrm{cm}$ corresponding well with the added amount of boron in the Si melt, assuming all boron atoms are electrically active. Despite the fact that they originate from two different casting runs, RGS_A and RGS_B materials show an average resistivity in the same range $(\sim 0.7 \Omega \mathrm{cm})$, demonstrating the reproducible resistivity of the novel RGS materials. On the other hand, the average resistivity of standard RGS material produced from the same melt as RGS_B material is $\sim 3.7 \Omega \mathrm{cm}$, indicating a lower 

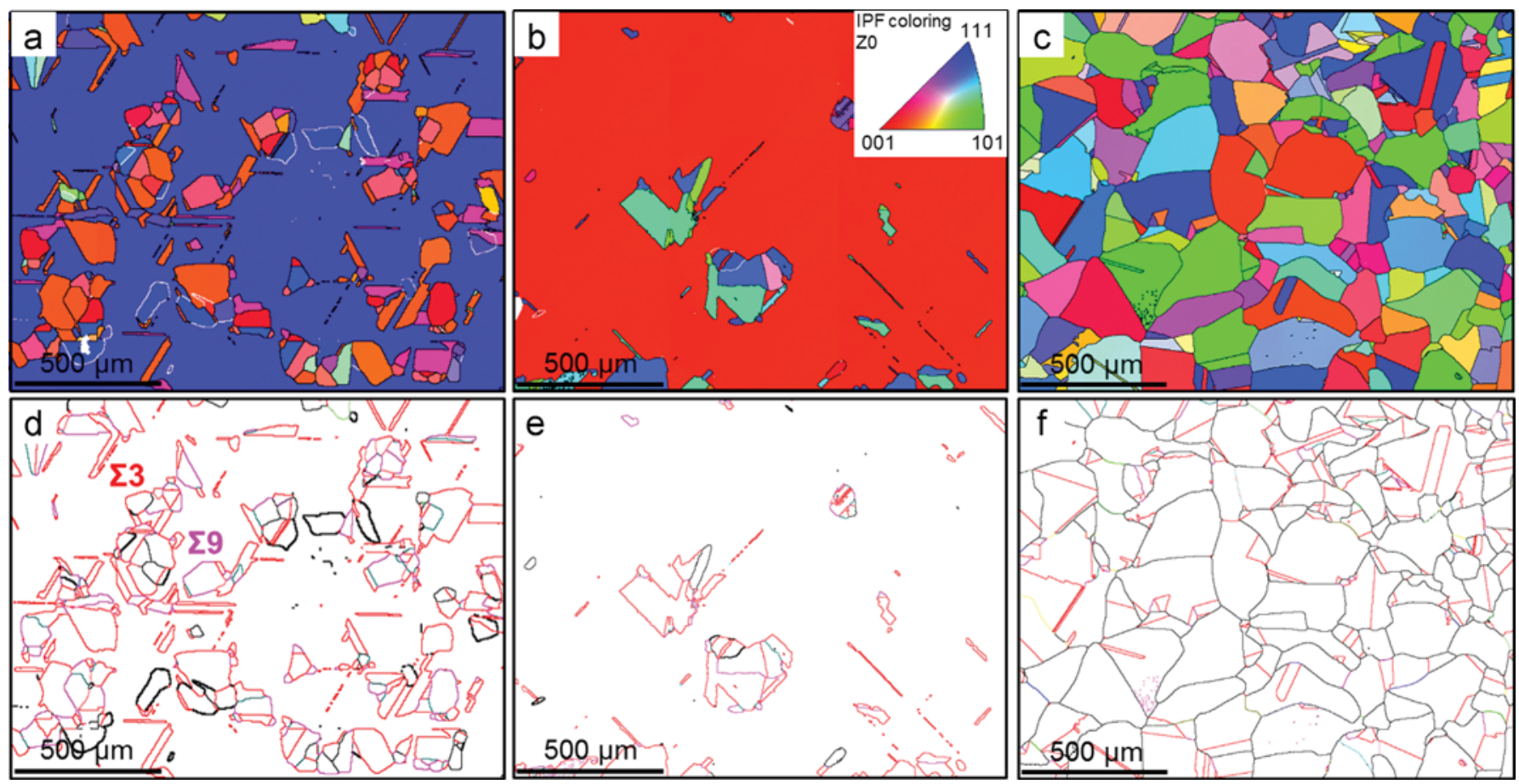

Fig. 2. On top the inverse pole figure (IPF) and grain boundaries (GBs) in a randomly selected area of the three investigated RGS materials are shown. The small angle $\left(<2^{\circ}\right.$ ) GBs are marked in white and the large angle $\left(>10^{\circ}\right) \mathrm{GBs}$ in black. For a clear illustration, the same area is shown on the bottom with only GBs. All coincident site lattice (CSL) GBs are highlighted in color and the random GBs are depicted in black, whereas the small angle $\left(<2^{\circ}\right)$ GBs are marked by thick black lines and the large angle $\left(>10^{\circ}\right)$ ones by fine black lines. From left to right: RGS A (a and d), RGS B (b and e) and RGS Std (c and f). For the two new RGS materials (left and center), most GBs are $\Sigma 3$ (shown in red) or $\Sigma 9$ (depicted in magenta), and large angle GBs are found only occasionally. On the other hand, for RGS_Std incoherent GBs dominate. (For interpretation of the references to color in this figure legend, the reader is referred to the web version of this article.)

boron activity [18]. This behavior is similar as for standard RGS material produced from earlier casting experiments. A detailed investigation of the activity of boron in the standard RGS material is beyond the scope of this contribution and will be presented in another paper.

The substitutional carbon concentration $\left[\mathrm{C}_{\mathrm{s}}\right]$ obtained via Fourier Transform Infra Red Spectroscopy (FTIR) is in the range of $(1.1 \pm 0.2) \cdot 10^{18} \mathrm{~cm}^{3}$ for the two novel materials and $(1.5 \pm 3.2)$. $10^{18} \mathrm{~cm}{ }^{3}$ for the standard one. Note that while $\left[C_{s}\right]$ is slightly lower for the new materials, the solid is still supersaturated with carbon (the solubility limit of the solid is about $5 \cdot 10^{17} \mathrm{~cm}^{3}$ [20]). The concentration of interstitial oxygen $\left[\mathrm{O}_{\mathrm{i}}\right]$ is comparable for the three RGS materials (in the range of $\left.(3.4 \pm 0.9) \cdot 10^{17} \mathrm{~cm}^{3}\right)$.

The wafer thicknesses at the beginning of solar cells processing were about $200 \mu \mathrm{m}, 210 \mu \mathrm{m}, 215 \mu \mathrm{m}$, and $225 \mu \mathrm{m}$ for $\mathrm{mc} \mathrm{Si}$, RGS_A, RGS_B, and RGS_Std, respectively.

\subsection{Solar cell process}

Fig. 3 shows the photolithography based laboratory cell process used [21]. The wafers were first laser cut into $5 \times 5 \mathrm{~cm}^{2}$, then the surface layers and the laser damage were removed by chemical polishing (CP). For mc Si reference wafers $\sim 10 \mu \mathrm{m}$ was removed from each side, whereas for RGS materials $\sim 25 \mu \mathrm{m}$ was etched away on each side to remove the defect rich layer [11]. To reduce reflectivity, a plasma texture was applied on the front side. The resulting texture is independent of crystal orientation and surface morphology and therefore suitable to compare the quality of all investigated materials. After a cleaning step in a PIRANHA solu tion, an emitter with $80100 \Omega / \square$ sheet resistivity was formed by $\mathrm{POCl}_{3}$ diffusion. The next step was deposition of an hydrogen rich amorphous silicon nitride $\left(\mathrm{SiN}_{x}: \mathrm{H}\right)$ passivation and anti reflection layer by Plasma Enhanced Chemical Vapour Deposition (PECVD) on the front side followed by a standard firing step in a belt

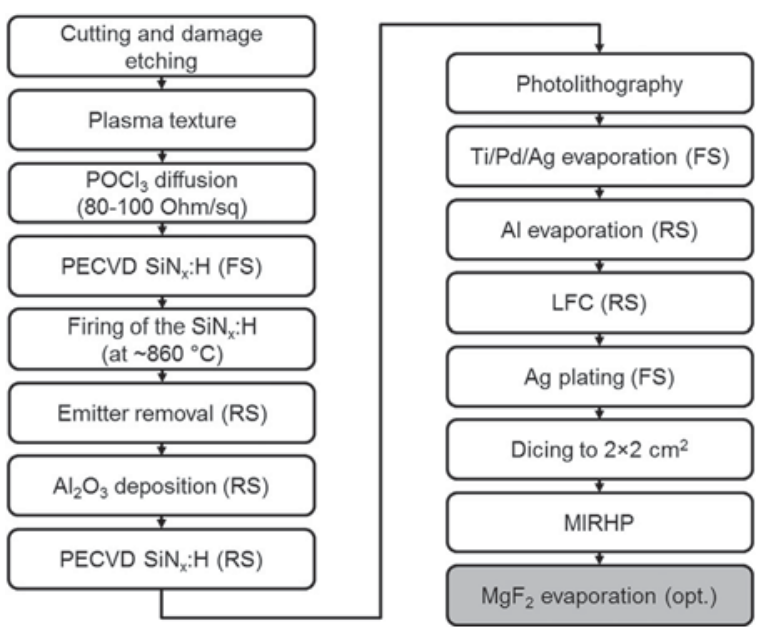

Fig. 3. Process flow chart of the photolithography-based solar cell process used in this work [21]. The last step (gray box) was not applied to all solar cells (i.e. thermal evaporation of a second layer anti-reflection coating (DARC) of $\mathrm{MgF}_{2}$ on the front side).

furnace for a few seconds, and wet chemical removal of the parasitic emitter on the rear side. Afterwards a Radio Corporation of America (RCA) cleaning was applied before the rear side pas sivation was realized by depositing an $\mathrm{Al}_{2} \mathrm{O}_{3} / \mathrm{SiN}_{x}: \mathrm{H}$ stack. The front side grid was structured via photolithography and a thin $\mathrm{Ti} /$ $\mathrm{Pd} / \mathrm{Ag}$ stack was evaporated as front contact seed layer. Subse quently, a $\sim 2 \mu \mathrm{m}$ thick Al layer was evaporated on the rear and local contacts were formed using the Laser Fired Contact (LFC) process [22]. After thickening the front side contact fingers by $\mathrm{Ag}$ Light Induced Plating (LIP), four $2 \times 2 \mathrm{~cm}^{2}$ solar cells were cut out of each $5 \times 5 \mathrm{~cm}^{2}$ wafer. Next, the solar cells were sintered under atomic hydrogen atmosphere using a Microwave Induced Remote 
Hydrogen Plasma (MIRHP) to anneal the $\mathrm{X}$ ray damage induced by metal evaporation via electron beam and to reduce the contact resistance. The last step (thermal evaporation of a second anti reflection coating of $\mathrm{MgF}_{2}$ ) is optional and was applied only to some solar cells. This cell process is particularly well suited for defect rich mc Si material, thanks to its low thermal budget, to the applied plasma texture, and to the excellent hydrogenation of bulk defects [21].

\section{Results and discussion}

Solar cells were first characterized by illuminated IV and spectral response measurements. Then material properties were investigated using spatially resolved measurement methods such as Light Beam Induced Current (LBIC), Electroluminescence (EL), and dark Lock In Thermography (dLIT). Finally, the quality of the material after phosphorus gettering and hydrogenation cell pro cessing steps was evaluated by mapping the minority charge car rier lifetime on one representative (etched) solar cell from each material group. Besides, infrared (IR) transmission microscopy analysis was performed after mechanical polishing of both wafer surfaces to investigate crystal defects and defect clusters.

\subsection{Electrical characterization of solar cells}

IV measurements were carried out under AM 1.5G illumination at $25{ }^{\circ} \mathrm{C}$ on solar cells made from the RGS materials presented above and from the block cast mc Si reference group. Fig. 4 shows box plots of short circuit current density $j_{\text {SC }}$ (Fig. 4a), open circuit voltage $V_{\mathrm{OC}}$ (Fig. 4b), fill factor FF (Fig. 4c), and cell efficiency (Fig. 4d). After first IV measurement (open symbols) a double layer anti reflection coating (DARC) of $\mathrm{MgF}_{2}$ was applied on the best solar cells of each group (closed symbols). As expected, applying a second layer anti reflection coating leads to a significant gain in $j_{\mathrm{SC}}$ for all materials, and to an increase in $V_{\mathrm{OC}}$ for all materials except the block cast mc Si reference. The deposition of the $\mathrm{MgF}_{2}$ layer on the block cast mc Si cells was carried out in another run than for the RGS cells, and we expect that the $V_{\mathrm{OC}}$ drop after DARC is due to handling issues. We conclude that for all materials, applying a DARC of $\mathrm{MgF}_{2}$ with an optimal thickness should lead to an increase of $0.60 .8 \%$ abs in efficiency, while FF remains constant.

For the standard RGS_Std and the novel RGS_B, $j_{\mathrm{SC}}$ is on the same level and higher than for RGS_A. This is most probably due to differences in material quality as will be confirmed in Section 3.4. We shall recall that RGS_B and RGS_Std were produced in the same casting experiment, while RGS_A comes from a different one. Indeed the material quality can differ drastically from experiment to experiment in the RGS lab type production tool [11]. Therefore, RGS_A material cannot be directly compared to RGS_B and RGS_Std.

Presumably for the same reason, $V_{\mathrm{OC}}$ is higher for RGS_Std than for RGS_A. Nevertheless, RGS_B presents $V_{\mathrm{OC}}$ values $\sim 10 \mathrm{mV}$ higher than RGS_Std. This is not only because of the lower resis tivity ( $0.7 \Omega \mathrm{cm}$ compared to $3.7 \Omega \mathrm{cm}$ for RGS_Std) but also because of the better material quality. Note that $V_{\mathrm{OC}}$ is lower for RGS_A than for RGS_Std $(3.7 \Omega \mathrm{cm})$, although its resistivity is in the same range as for RGS_B $(0.7 \Omega \mathrm{cm})$. For the novel material RGS_B, $V_{\mathrm{OC}}$ values as high as $608 \mathrm{mV}$ could be achieved, up to now the highest obtained for RGS material. For comparison, block cast mc Si reference cells show $V_{\mathrm{OC}}$ values up to $630 \mathrm{mV}$.

FF values below $72 \%$ for RGS_Std solar cells are typical for standard RGS material [11]. Interestingly, FFs of solar cells made from the novel RGS materials are almost all above $78 \%$ and on the same level as for the block cast mc Si cells. These high FFs are the highest obtained for RGS materials to date. This is a hint that the novel RGS materials do not suffer from the shunting problem usually observed for standard RGS material [13]. In addition, the distribution of FF values for RGS_A and RGS_B is remarkably tighter than for RGS_Std. Together with the smaller spread in $j_{\mathrm{SC}}$
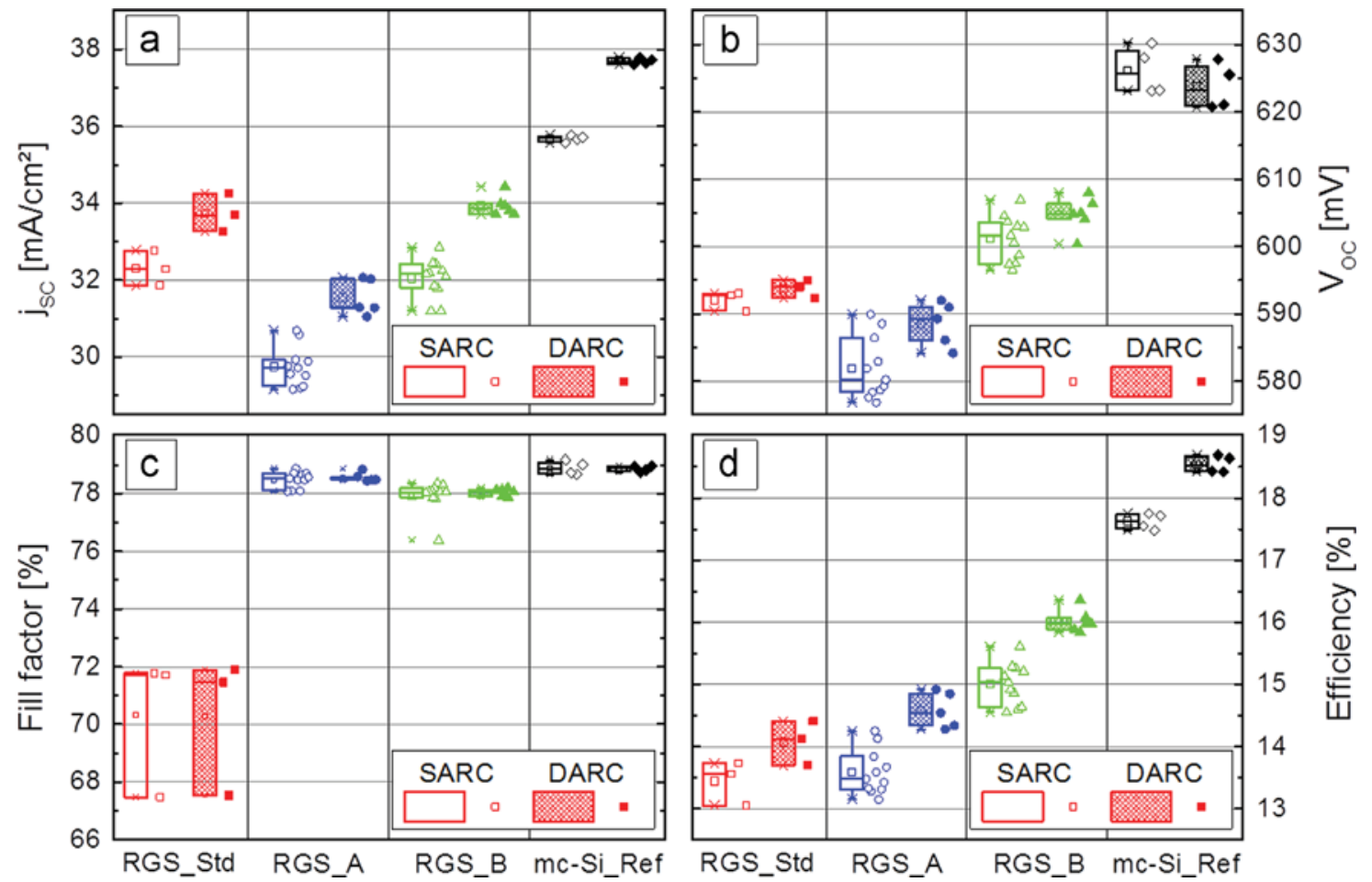

Fig. 4. Box plots of solar cell parameters extracted from illuminated IV measurements of the three investigated RGS materials and of block-cast mc-Si references. Open symbols and open boxes represent values for solar cells with $\mathrm{SiN}_{x}: \mathrm{H}$ single layer anti-reflection coating (SARC). The best cells of each group received a second layer antireflection coating (DARC) of $\mathrm{MgF}_{2}$ (filled symbols and dense checkered boxes). 
and $V_{\mathrm{OC}}$, this may reflect the better homogeneity of the two new materials compared to the standard one.

RGS_A and RGS_B materials resulted in higher efficiencies than RGS_Std because of the higher FF values. In particular, RGS_B solar cells show significant improvement compared to the other two groups. Note again that only RGS_B can be directly compared to RGS_Std because it originates from the same casting run. Record efficiencies above $16 \%$ have been achieved (one cell whose IV and power curve are shown in Fig. 5 was certified at Fraunhofer ISE CalLab to $16.1 \%)$. This represents a significant progress compared to the former efficiency record of $14.4 \%$ for RGS material [17].

Fig. 6 shows series resistance $R_{\mathrm{S}}$, recombination/diffusion cur rent density of the bulk (emitter and base) $j_{01}$, parallel resistance $R_{\mathrm{P}}$, and recombination current density in the space charge region $j_{02}$. These values were obtained by fitting the illuminated IV curves with the two diode model, assuming a planar two dimensional emitter structure. We note that for all processed solar cells, $R_{\mathrm{S}}$ is $<0.6 \Omega \mathrm{cm}^{2}$ indicating a successful metallization of the cells.

Referring to Fig. 6a, we now first discuss the $j_{01}$ and the $R_{\mathrm{S}}$ results for RGS_A, RGS_B and $\mathrm{mc}$ Si_Ref and then analyze the unexpected trends of these parameters for RGS_Std.

The emitter contribution $j_{0 \mathrm{e}}$ to $j_{01}\left(j_{01}=j_{0 \mathrm{e}}+j_{0 \mathrm{~b}}, j_{0 \mathrm{e}}\right.$ the emitter and $j_{\mathrm{ob}}$ the base contribution) can be neglected due to the sig nificantly higher $j_{0 \mathrm{~b}}$ contribution in these defect rich materials. Therefore, $j_{01}$ can be approximated by $j_{01} \approx j_{0 \mathrm{~b}}=n_{\mathrm{i}}^{2} e D_{\mathrm{e}} / N_{\mathrm{A}} L_{\mathrm{eff}}$, where $n_{\mathrm{i}}$ is the intrinsic carrier concentration, $e$ the electron charge, $D_{\mathrm{e}}$ the electron diffusion constant, $N_{\mathrm{A}}$ the acceptor concentration and $L_{\text {eff }}$ the effective diffusion length of electrons (the materials are p doped) [23,24]. Thus $1 / j_{01}$ is proportional to $L_{\text {eff }}$ and can be seen as a measure of the bulk quality. Indeed, RGS_Std left aside, $j_{01}$ is highest for RGS_A and lowest for the mc Si_Ref, correlating well with the opposite trends for $j_{\mathrm{SC}}$ and $V_{\mathrm{OC}}$ (see Fig. 4).

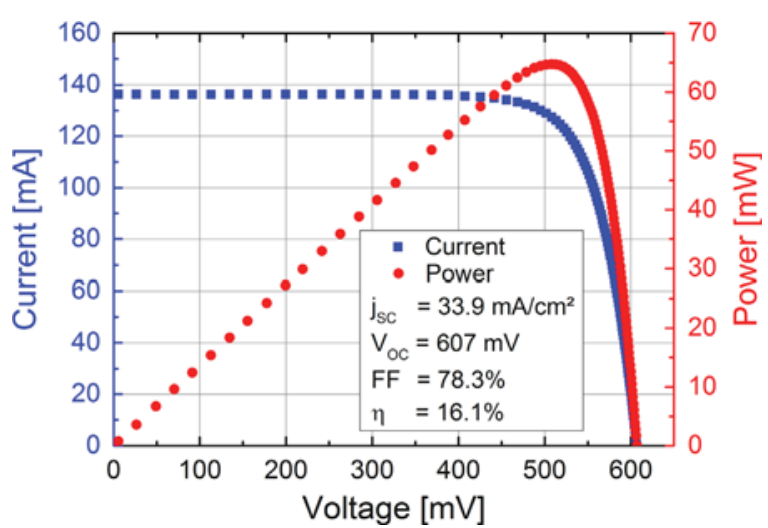

Fig. 5. Current and power as a function of voltage for one solar cell of group RGS_B. The measurement was performed at Fraunhofer ISE CalLab, measured cell area is $4.02 \mathrm{~cm}^{2}$.

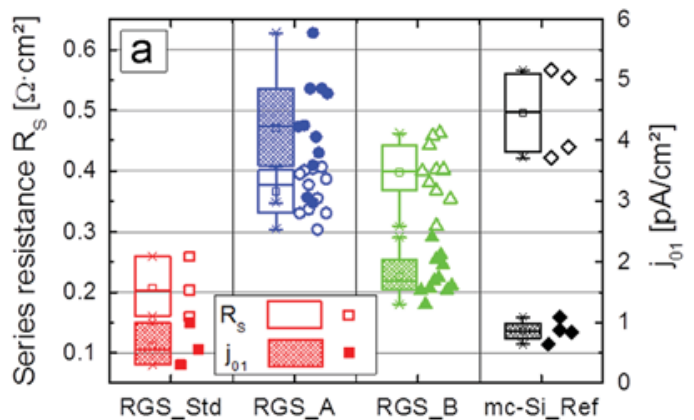

Regarding $R_{\mathrm{S}}$, values around $0.5 \Omega \mathrm{cm}^{2}$ for RGS_A, RGS_B and mc Si_Ref are found which are realistic.

We turn now to RGS_Std. The unexpectedly low $R_{\mathrm{S}}$ values (in the range of $0.2 \Omega \mathrm{cm}^{2}$ ) are most likely erroneous because we expect that for this material current density $j$ is overestimated. In Sections 3.3 and 3.4 we will show that at some localized regions of RGS_Std cells $j_{\mathrm{SC}}$ is significantly higher while in the same areas $L_{\mathrm{diff}}$ is lower. In previous research a local increase in $L_{\text {eff }}$ without an increase in $L_{\text {diff }}$ was interpreted as the result of current collecting structures forming a three dimensional emitter extension into the Si bulk [7]. As will be shown in Sections 3.3 and 3.4, the regions with current collecting structures can represent a significant area fraction of RGS_Std cells. Since the two diode model does not take into account the locally higher $j_{01}$ at these regions, the average $j_{01}$ is underestimated and unrealistically lower than the typical $1 \mathrm{pA} /$ $\mathrm{cm}^{2}$ value for block cast mc Si [23]. To get a better understanding of this behavior, a spatially resolved image of $j_{01}$ and the analysis of the local solar cell properties such as described in [25 28] would be necessary, but this is not the focus of this contribution.

Finally, we discuss the fit results of $j_{02}$ and $R_{\mathrm{P}}$ (Fig. 6b). For RGS_Std the average $j_{02}$ is above $20 \cdot 10{ }^{8} \mathrm{~A} / \mathrm{cm}^{2}$, i.e. at least four times higher than for the other materials which show $j_{02}$ values around $5 \cdot 10{ }^{8} \mathrm{~A} / \mathrm{cm}^{2}$ for RGS_B or below for RGS_A and $\mathrm{mc}$ Si_Ref. The higher $j_{02}$ for RGS_Std correlates well with lower $R_{\mathrm{P}}$ (below $1 \mathrm{k} \Omega \mathrm{cm}^{2}$ ) as already shown by Hess et al. [11]. On the contrary, all solar cells from the two novel RGS materials and from the block cast mc Si have $R_{\mathrm{P}}>10 \mathrm{k} \Omega \mathrm{cm}^{2}$, i.e. at least an order of magnitude higher than for RGS_Std. Note that the high $\mathrm{j}_{02}$ corre lates well with the low $R_{\mathrm{p}}$ and low FF, see Fig. 4c.

\subsection{Spectral response and reflectivity measurements}

Spectral response was measured on one representative cell of each group selected in a way that its efficiency is close to the median value of the group. Fig. 7 shows the Internal Quantum Efficiency (IQE) as function of the wavelength. Below $500 \mathrm{~nm}$ the four IQE curves are similar, the values for the mc Si solar cells being only slightly higher. This indicates that front surfaces and emitters of the four measured solar cells are comparable. However, there is a clear difference between the four materials at wave lengths above $500 \mathrm{~nm}$ : the IQE of the block cast mc Si solar cell is significantly higher than for RGS_B and RGS_Std, these RGS materials having higher IQE than RGS_A, reflecting the trend observed for $j_{\text {SC }}$ (see Fig. 4a). RGS_Std and RGS_B show similar values except for the 'hump' observed between 950 and $1100 \mathrm{~nm}$ for RGS_B. This behavior can at least partly be explained by the increased reflectivity of RGS_B in this wavelength interval because of the thinner cell ( $170 \mu \mathrm{m}$ compared to $200 \mu \mathrm{m}$ for RGS_Std).

The reflectivity of the block cast mc Si solar cell shows lower values than for RGS materials and two minima because only the mc Si solar cell received a double layer anti reflection coating. Note that the second layer anti reflection coating should not have

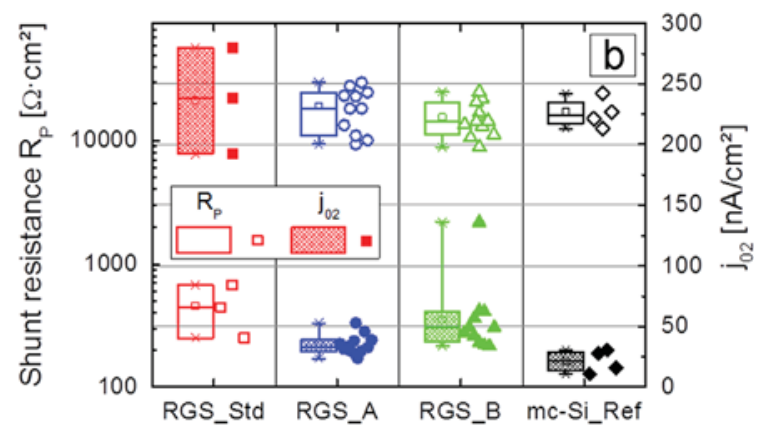

Fig. 6. (a) $R_{\mathrm{S}}$ (open symbols) and $j_{01}$ (filled symbols) and (b) $R_{\mathrm{P}}$ (open symbols) and $j_{02}$ (filled symbols) obtained from the two-diode model fits of the illuminated IV curves. 
any influence on the IQE. For all RGS materials the reflectivity curves are similar up to $1000 \mathrm{~nm}$. Between $1000 \mathrm{~nm}$ and $1200 \mathrm{~nm}$ the reflectivity of the novel RGS materials is higher than for RGS_Std. The smaller cell thickness for the new RGS materials can explain this difference at long wavelengths. Note that the minima of these reflection curves are different, indicating slight variations of the anti reflection coating thickness from cell to cell.

The effective diffusion length $L_{\text {eff }}$ was derived by fitting the IQE curves by the procedure proposed by Basore [24] (IQE ${ }^{1}\left(L_{\alpha}\right)=$ $1+L_{\alpha} / L_{\text {eff }}, L_{\alpha}$ is the absorption length) and is shown in the caption of Fig. 7. For RGS_B and RGS_Std, $L_{\text {eff }}$ is in the same range as previously measured on standard RGS material [13] and two times higher than for RGS_A. This reflects the lower material quality of RGS_A compared to RGS_B and RGS_Std, most probably because of the different casting experiments as discussed above. Because the diffusion length of the RGS solar cells is less than half their

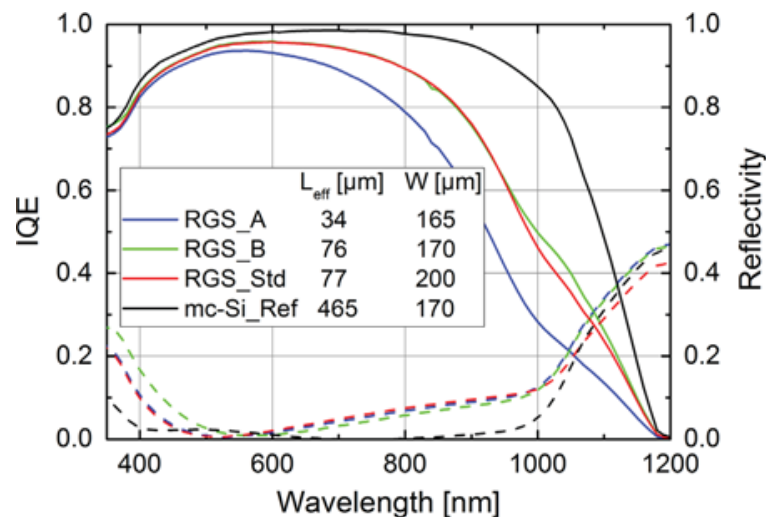

Fig. 7. IQE (solid lines) and reflectivity (dashed lines) of the investigated materials. $\mathrm{mc}$-Si_Ref was measured after deposition of the second anti-reflection coating of $\mathrm{MgF}_{2}$, hence its reflection curve has two minima. The measured cell thickness $W$ and the extracted $L_{\text {eff }}$ values are shown in the legend. thickness, they do not benefit significantly from the advanced rear side passivation of the PERC cell process, in contrast to the solar cells made from block cast mc Si having diffusion lengths of about three times the wafer thickness. A reduction of the RGS wafer thickness to values comparable with $L_{\text {eff }}$ may induce a further increase of the solar cell efficiency, provided suitable light trapping results in similar charge carrier density generation.

\subsection{Spatially resolved investigations: comparison of LBIC, EL and dLIT maps}

LBIC and EL maps of the same representative RGS cells are shown on Fig. 8. First we note that the maps of the novel materials are more homogeneous than those of the standard one, indicating better material homogeneity. For the novel materials RGS_A (Fig. 8a and d) and RGS_B (Fig. 8b and e), the spatial distribution of IQE (as estimated from the LBIC map) and of the EL signals are similar, i.e., areas 1 and 2 with increased IQE values also show high intensities in the corresponding EL image. The comparable spatial distribution of the IQE and EL maps translates into a similar shape of the histograms at the right of each map. In contrast, for RGS_Std the IQE map (Fig. 8c) differs significantly from the EL map (Fig. 8f). For example, the encircled region 3 shows a high IQE (IQE > 0.7), but an EL intensity lower than average. EL measures band to band radiation which is related to the local diffusion length $L_{\text {diff }}$ and therefore reflects the material quality while the LBIC measurement technique actually measures $j_{S C}$ at a given wavelength connected to the external quantum efficiency (EQE) and, taking reflectivity into account, to IQE. Thus region 3 for RGS_Std shows high IQE and $j_{\text {SC }}$ although material quality is low. Earlier research on standard RGS material concluded that such regions with higher $j_{\mathrm{SC}}$ and lower material quality have current collecting structures $[7,13]$.

To better visualize the difference between the new and the standard RGS materials, the data of Fig. 8 is plotted in 3D dis tribution maps of IQE and of EL intensity (see Fig. 9) using the
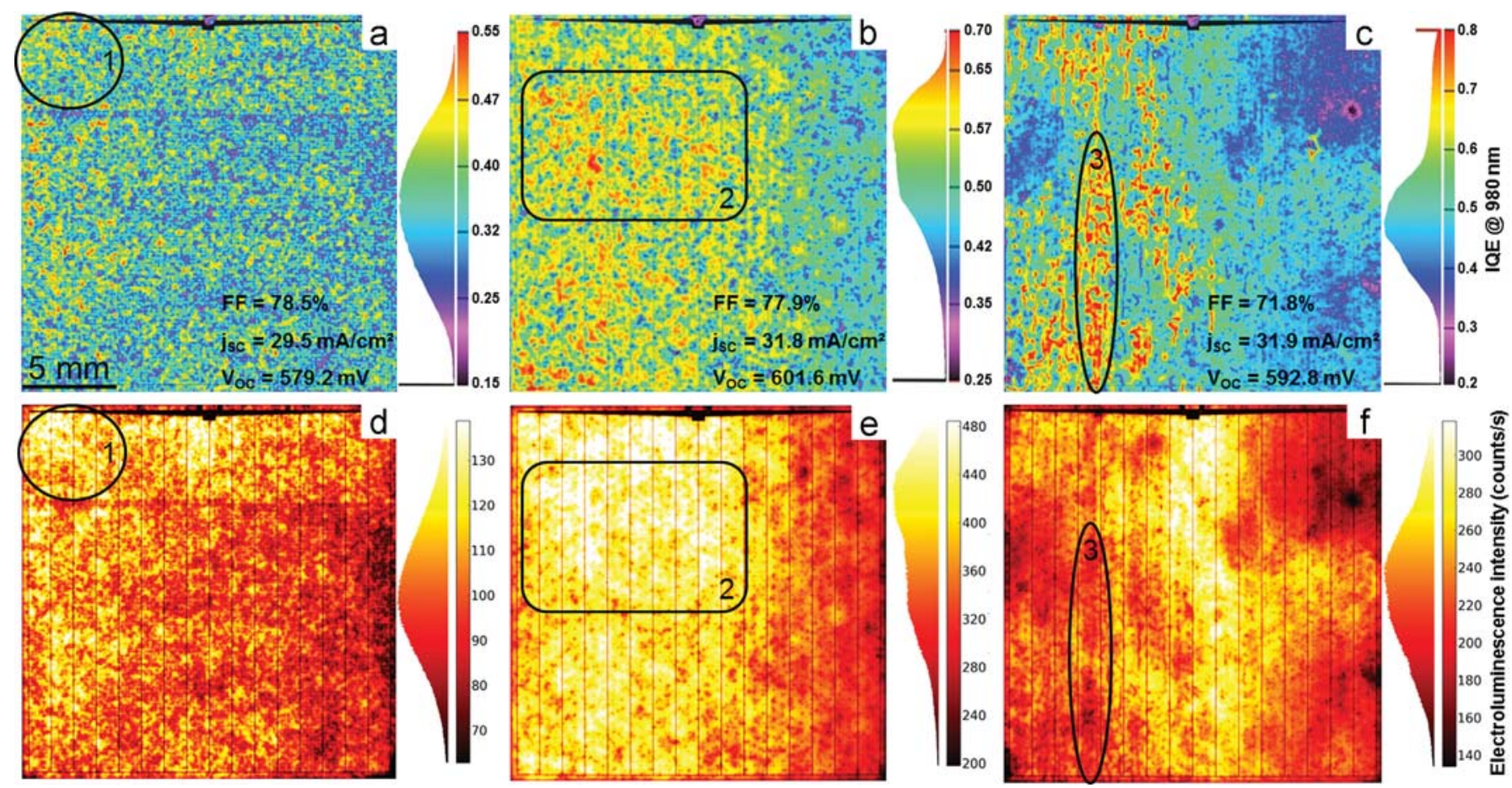

Fig. 8. Comparison between IQE maps at $980 \mathrm{~nm}$ wavelength and $25 \mu \mathrm{m}$ spatial resolution (top), and corresponding EL maps (bottom) of the three same representative RGS cells as measured in Fig. 7. From left to right: RGS_A, RGS_B, RGS_Std. The EL maps have a pixel resolution of about $20 \mu \mathrm{m}$ and all EL measurements were made under the same conditions. Note that the maps are differently scaled to highlight the different features discussed in the text. 
procedure given in Appendix A. For RGS_A and RGS_B solar cells (Fig. 9a and b), the IQE is approximately proportional to EL intensity whereas for RGS_Std, the points having IQE above 0.6 (within the red bounded area in Fig. 9c) clearly deviate from the proportionality trend. Precisely these points correspond to the regions with current collecting structures such as region 3 in Fig. 8. Therefore, we conclude that for RGS_Std IQE values well above 0.6 are caused by current collecting structures increasing $j_{\mathrm{SC}}$ and the LBIC signal in these regions.

Fig. 10 shows dark Lock In Thermograms (dLIT) of the same solar cells taken by applying different forward and reverse biases. Solar cells made from the new RGS materials RGS_A (left column) and RGS_B (middle column) show significant signal intensity under forward bias of $0.53 \mathrm{~V}$ and under large reverse bias of $7.8 \mathrm{~V}$, while a low reverse bias of $0.96 \mathrm{~V}$ does not generate a significant thermal signal. This is a hint that these two solar cells have shunts with nonlinear (diode like) characteristic [29,30]. According to previous research, this type of shunt is not detrimental to solar cell performance [29] as confirmed in our case by the FF values of about 78\% for these cells (78.5\% for RGS_A and 77.9\% for RGS_B), i.e. close to the values of the mc Si references (see Fig. 4). For the RGS_A solar cell (left column), three bright spots are visible on the right edge region of the images taken at $0.53 \mathrm{~V}$ forward and $7.8 \mathrm{~V}$ reverse bias. These nonlinear edge shunts are most probably caused by sawing during edge isolation. Surprisingly, for the RGS_B solar cell the dLIT image taken at $7.8 \mathrm{~V}$ reverse bias looks different to the forward bias thermogram. This is probably the result of nonlinear field induced shunts as discussed in $[29,30]$.

Large areas of the RGS_Std solar cell show bright contrast under $0.53 \mathrm{~V}$ forward bias as well as under $0.96 \mathrm{~V}$ and $6.8 \mathrm{~V}$ reverse
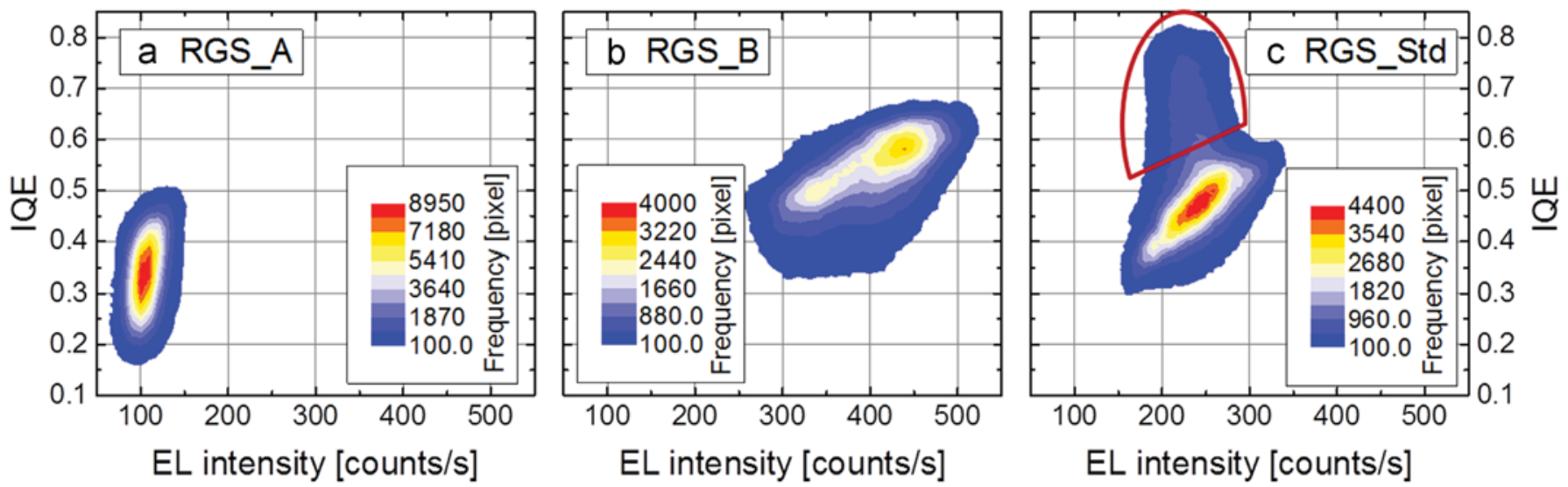

Fig. 9. Distribution maps of IQE as function of EL intensity for the data of Fig. 8. The color coding corresponds to the pixel count frequency for a given \{EL intensity; IQE $\}$ set. Note that the maximum of the color scaling is different for the three graphs.
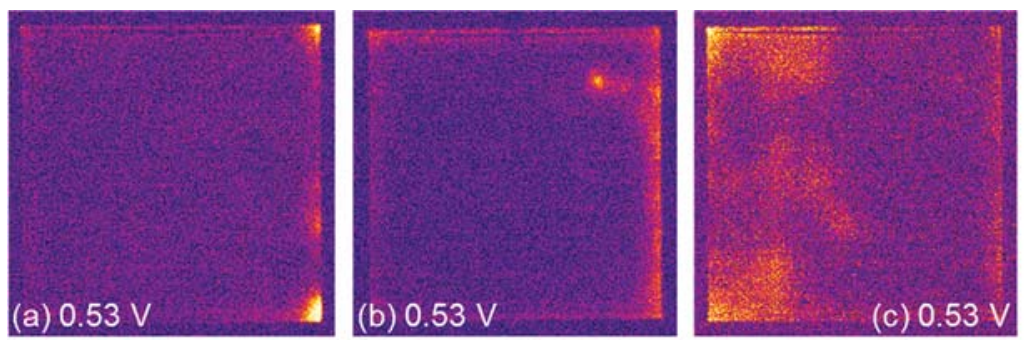

high
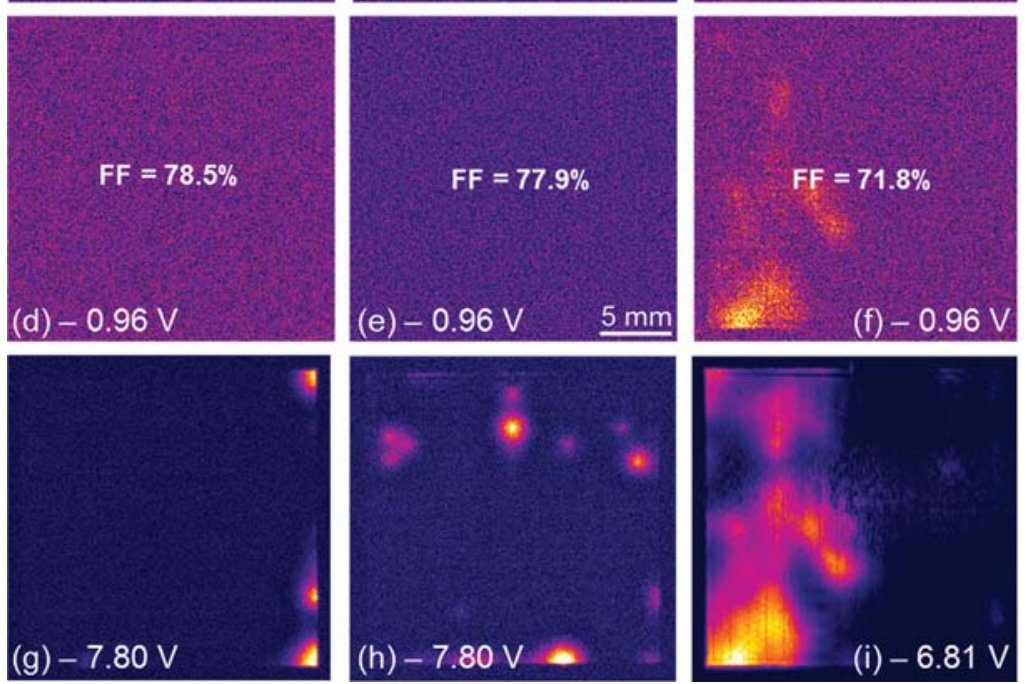

Fig. 10. dLIT measurements of the same solar cells as in Figs. 7-9: RGS_A (left), RGS_B (center), and RGS_Std (right). The top row shows images acquired at a forward bias of $0.53 \mathrm{~V}$, the rows below are taken at a reverse bias of $-0.96 \mathrm{~V}$ (middle) and $-7.80 \mathrm{~V}$ (bottom). Measurements were carried out with a lock-in frequency of $45 \mathrm{~Hz}$ and an acquisition time of $10 \mathrm{~min}$. Note that all images are differently scaled. 
bias. At the upper left corner a thermal signal is visible at forward bias and reverse bias of $6.8 \mathrm{~V}$ but not at lower reverse bias of $0.96 \mathrm{~V}$. The bright areas visible in the dLIT images under forward and low reverse bias result from shunts having a linear (ohmic) IV characteristic. Fig. 11 shows the dLIT thermogram at $6.8 \mathrm{~V}$ reverse bias overlaid with the corresponding IQE map. It shows that the regions with higher brightness in the lock in thermograms (the shunted regions) correlate well with the regions with high LBIC signal (the regions with current collecting structure). We conclude that the current collecting structures cause ohmic shunts to the investigated cell resulting in a FF significantly lower $(71.8 \%$ for this cell) than that of the other materials.

\subsection{Measurement of bulk lifetime}

Lifetime samples were prepared from the same solar cells to gain direct insight into the bulk quality without having to take into account the effect of the current collecting structures on charge transport processes and on the diffusion length. To prepare the samples from the solar cell, first the contacts were etched in aqua regia, then the passivation layers $\left(\mathrm{SiN}_{x}: \mathrm{H}\right.$ on the front and $\left.\mathrm{Al}_{2} \mathrm{O}_{3}\right)$ $\mathrm{SiN}_{\mathrm{x}}: \mathrm{H}$ on the rear) were etched in diluted $\mathrm{HF}$ and finally the emitter was removed using a CP etch. After a RCA cleaning step the surfaces were passivated by deposition of a $\sim 30 \mathrm{~nm}$ thick $\mathrm{Al}_{2} \mathrm{O}_{3}$

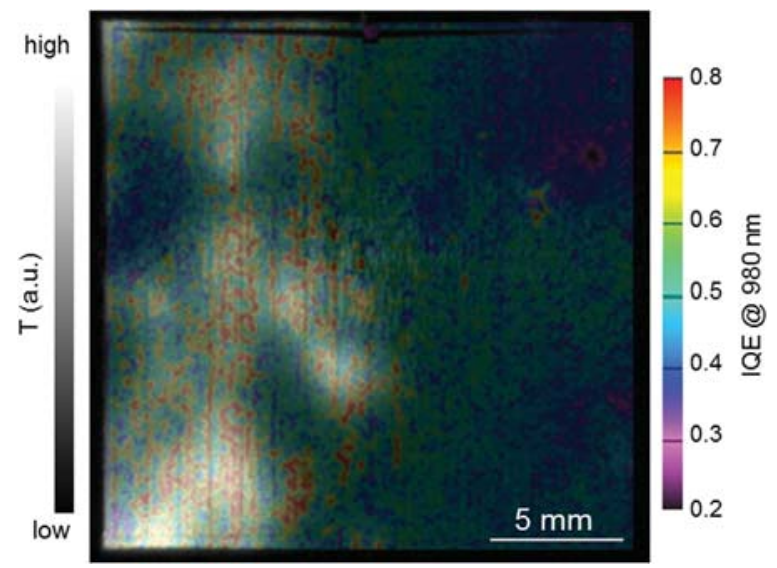

Fig. 11. Overlay of the grayscale dLIT thermogram of the RGS_Std solar cell taken at $-6.8 \mathrm{~V}$ reverse bias (Fig. 10i) and the corresponding IQE map (Fig. 8c). Despite the lower spatial resolution of the dLIT image compared to that of the IQE map, the bright spots on the lock-in thermogram correlate well with the regions of (artificially) high IQE (IQE $>0.6)$. using plasma assisted Atomic Layer Deposition (ALD) and the passivation was activated under $\sim 10$ mbar $\mathrm{N}_{2}$ atmosphere at $\sim 420^{\circ} \mathrm{C}$ for $30 \mathrm{~min}$. Spatially resolved lifetime measurements were carried out by Microwave detected Photo Conductance Decay ( $\mu$ PCD) on a Semilab WT 2000 and are shown in Fig. 12. As expected from the analysis of the IV characteristic (Section 3.1), RGS_A (Fig. 12a) shows the lowest lifetime values, most probably because of the different casting experiment in contrast to RGS_B and RGS_Std. For RGS_Std the arithmetic mean (average) lifetime is $10.2 \mu \mathrm{s}$ and the harmonic mean is $8.8 \mu$ s, i.e. in the range of values already measured for standard RGS material in previous work [13]. This shows that this sample is representative of stan dard RGS material. In contrast, the arithmetic and the harmonic mean lifetime values of RGS_B (Fig. 12b) are about two times higher than for standard RGS (Fig. 12c), demonstrating the pro mising quality of the new material.

The relative discrepancy between the arithmetic and the har monic mean values of the minority carrier lifetime $\left(\tau_{\mathrm{a}} \tau_{\mathrm{h}}\right) \times 100 / \tau_{\mathrm{a}}$ is a gauge for the homogeneity of the material quality. These rela tive discrepancies are 2.8\%,6.4\% and 13.7\% for wafers RGS_A, RGS_B, and RGS_Std, respectively. Again we conclude that the two novel RGS materials are more homogeneous than the standard one.

For RGS_Std the lifetime distribution (Fig. 12c) is similar to the EL intensity distribution (Fig. 8f). Likewise we find areas like region 3 with low lifetime and with IQE well above 0.6 (see Fig. 8c) because of the high effective diffusion length. Such discrepancy between IQE (or rather $L_{\text {eff }}$ ) map and lifetime map was also found in the past on standard RGS material and was attributed to the current collecting structures by Hahn et al. [7]. In contrast, for the new RGS materials, areas with high lifetime have also increased IQE values and show high intensity in the EL image (e.g., encircled regions 1 and 2 in Figs. 8 and 12). We confirm here our previous conclusion with a more direct measurement of the material quality.

\subsection{Infrared transmission microscopy investigation}

In the previous section we showed that in contrast to the new RGS materials, the standard RGS material suffers from shunting issues due to current collecting structures at regions of low material quality. In this section we present IR microscopy inves tigations aiming at making the first steps towards understanding the reason for the absence of current collecting structures in the new materials. Silicon has a bandgap of $\sim 1.1 \mathrm{eV}$ and is therefore transparent for IR light (with energy below $1.1 \mathrm{eV}$ ) at room tem perature. However, extended crystal defects such as precipitates,
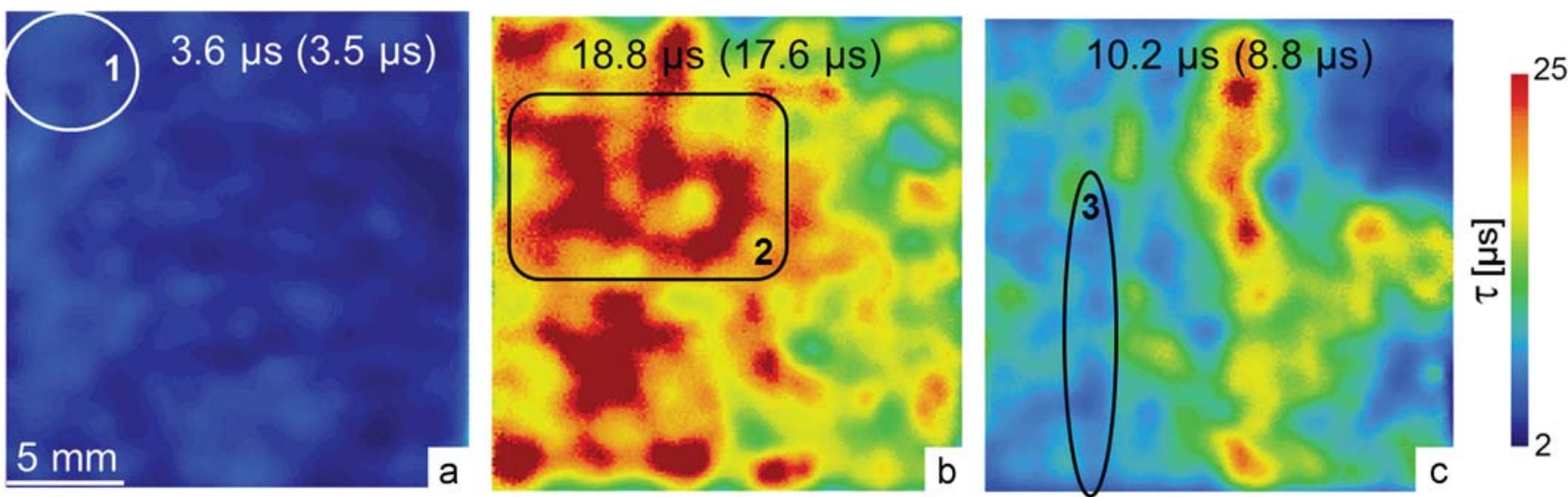

Fig. 12. $\mu$-PCD lifetime maps of samples from the same solar cells measured in Figs. 7-10: (a) RGS_A, (b) RGS_B and (c) RGS_Std. The arithmetic mean is written on top with the corresponding harmonic mean in brackets. 

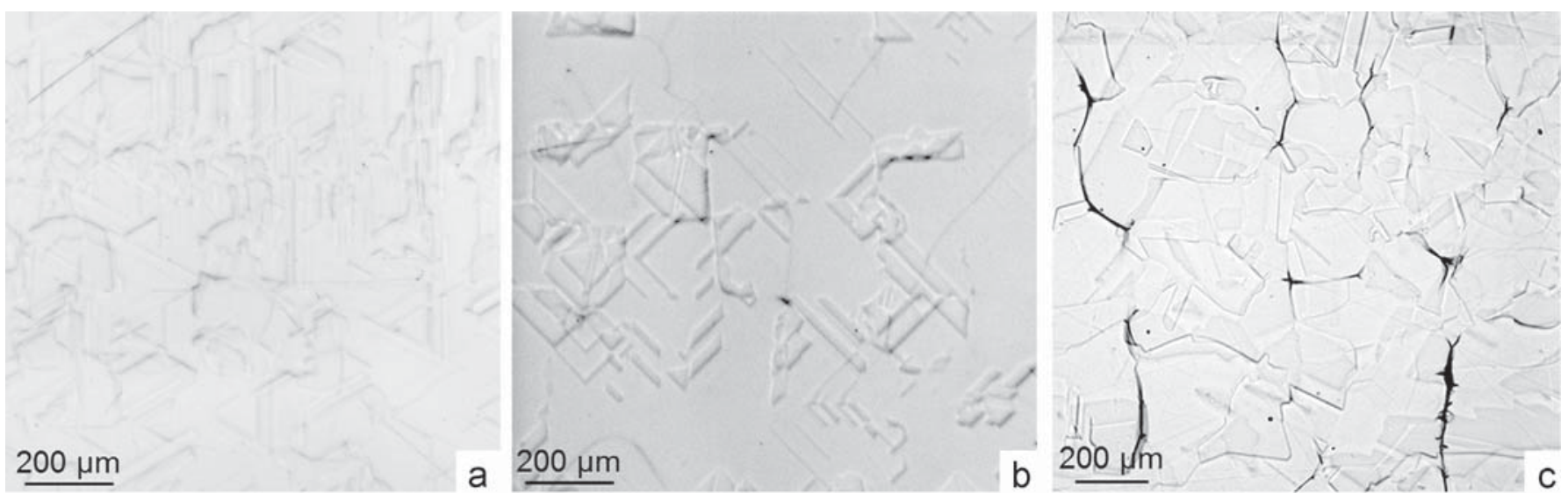

Fig. 13. IR microscopy images of randomly selected regions of the three investigated RGS materials: (a) RGS_A, (b) RGS_B, (c) RGS_Std. The dark features on RGS_Std indicate the presence of current collecting structures as also found by Hess et al. [31]. Some grain boundaries are visible because of the mildly alkaline etch character of the polishing solution used.

micro cracks and grain boundaries can scatter and absorb IR light and thus create a shadowy or dark contrast in the image [31].

After removing the passivation layer from the lifetime samples shown in Fig. 12, both sides were mechanically polished and samples were investigated by IR transmission microscopy. Ran domly selected regions from the three investigated RGS materials are presented in Fig. 13. Some grain boundaries are visible as the result of preferential etching of the mildly alkaline polishing solution. Other optical surface artifacts are a few dark points visible on some pictures (due to dust particles on the surface or residues of the resin used to fix the samples during polishing) or scratches due to polishing. Only such surface artifacts are visible on the IR images of the two new RGS materials (Fig. 13a and b). On the other hand, for RGS_Std (Fig. 13c) dark patterns are observed along some grain boundaries over the whole image. Fig. 13c shows that the 'dark grain boundaries' are orientated approximately parallel to each other, with a typical spacing of a few $100 \mu \mathrm{m}$ which corresponds to the typical distance between two adjacent nucleation sites. As was demonstrated in a previous study, these grain boundaries are typically the large angle grain boundaries corresponding to the impingement of neighboring crystals, and where the segregated carbon generates $\mathrm{SiC}$ nucleation and fila ment growth [12].

In a previous study of a comparable standard RGS material Hess et al. showed that the positions of such 'dark grain boundaries' correlated with regions of IQE values well above 0.6, that these grain boundaries were electrically active and contained $\mathrm{SiC}$ pre cipitates [13]. This study concluded that these current collecting structures are responsible for the $\mathrm{FF}$ values below $72 \%$, i.e. the shunting is caused by conductive SiC filaments.

The absence of 'dark grain boundaries' on the new RGS mate rial as well as the absence of current collecting structures as concluded from the previous sections suggest that conductive $\mathrm{SiC}$ filaments are absent in the new RGS materials. Nevertheless, the new materials are supersaturated with carbon, i.e. the carbon is present in a metastable state.

EBSD measurements (Fig. 2) showed that in the new materials the grain boundaries are mostly of $\Sigma 3$ and $\Sigma 9$ nature, and large angle (incoherent) grain boundaries are very rarely observed. Thus, a fundamentally different characteristic of the new material is that crystal orientation control during the initial stages of growth prevented the formation of large angle grain boundaries when adjacent nucleated crystals met.

An important question is the effect of the grain boundary coherency on the nucleation potency and growth of SiC crystals. In general, precipitate nucleation is more favorable at grain boundaries because of the decrease of the energy of the system in doing so [32,33]. However, $\Sigma 3$ twin boundaries are low energy coherent 2D defects (the atoms do not have dangling bonds). As a consequence, their stacking fault energy (about $2670 \mathrm{~mJ} \mathrm{~m}^{2}$ [34]) is about one order of magnitude lower as the energy of an incoherent grain boundary (in the range of $5001000 \mathrm{~mJ} \mathrm{~m}^{2}$ [34]). Thus the nucleation site potency of twins for $\mathrm{SiC}$ precipitates can be expected to be significantly lower than that of incoherent grain boundaries.

Based on this hypothesis, research is being done to evaluate the effect of controlling the crystal orientation on the growth mor phology, on the carbon segregation, on its incorporation in the crystal and on SiC nucleation and growth under RGS conditions.

\section{Conclusion}

Two novel RGS materials were produced using differently oriented monocrystalline silicon substrates, resulting in pre dominantly (111) or (100) oriented quasi monocrystalline RGS materials [18]. The new materials have been processed into solar cells and compared to standard RGS material. The new RGS material shows a resistivity value $(0.7 \Omega \mathrm{cm})$ correlating well with the amount of boron added in the silicon melt in contrast to the standard RGS material where not all added boron is electrically active. The predominant orientations offer the possibility to use alkaline texture.

In contrast to standard RGS solar cells, solar cells processed from the new RGS material show no material induced linear shunts because current collecting structures are absent. Although the (111) oriented RGS_A material originates from another crys tallization run as the (100) oriented RGS_B, both materials crys tallized on monocrystalline silicon substrates do not show any presence of current collecting channels in contrary to the standard RGS material RGS_Std. Furthermore, spatially resolved measure ments reveal that the bulk quality of the two new RGS materials is more homogeneous than those of the standard one. This was demonstrated by comparing LBIC maps, $\mu$ PCD lifetime maps and EL images, by dLIT measurements and finally by IR transmission microscopy investigations. Consequently, for the novel RGS materials FF, $R_{\mathrm{P}}$ and $j_{02}$ are in the same range as for the block cast mc Si reference material. Due to the FF values above $78 \%$, solar cell efficiencies of the new RGS materials are significantly higher than those of standard RGS material. For RGS_B, $V_{\mathrm{OC}}$ values up to $608 \mathrm{mV}$ and efficiencies $>16 \%$ could be achieved due to the higher material quality: the lifetime after processing was $\sim 19 \mu \mathrm{s}$, 
i.e. twice as high as for standard RGS material. This is a significant improvement of the previous efficiency record of $14.4 \%$ for RGS material [17]. The absence of current collecting structures and the different dopant activity of the new materials compared to stan dard material produced from the same melt point towards sig nificant differences in the solidification behavior.

\section{Acknowledgments}

We like to thank L. Mahlstaedt, F. Mutter, and C. Paape for their assistance during sample preparation. Part of this work was finan cially supported by the German Federal Ministry for the Environ ment, Nature Conservation and Nuclear Safety (FKZ0325581) and within the research cluster 'SolarWinS' (FKZ0325270F). The supply of RGS wafers by RGS Development B.V. is gratefully acknowledged. The content is the responsibility of the authors.

\section{Appendix A}

The graphs shown in Fig. 9 were obtained aligning the IQE topogram and the EL map of each solar cell so that the corre sponding areas of the two images match each other. Using the program Matlab, the raw data was first transformed into a data format importable by the program Avizo Fire 8.0.1 [35] which is appropriate for the analysis of 3 dimensional image data. With Avizo the spatial resolution of the EL maps $\sim 20 \mu \mathrm{m}$ was first interpolated to $25 \mu \mathrm{m}$, the one of the IQE topograms. Afterwards the two images were adjusted referring to the busbars, so that the busbar regions of each image match very well those of the other. Subsequently, the data of the edges and the busbar regions were removed in order to reduce their possible negative effects on the statistics. The finally obtained image data with $751 \times 718$ pixels were then imported into Matlab and hence given out as a matrix.

\section{References}

[1] P. Fath, A. Ramakrishnan, N. Rösch, W. Herbst, Cost reduction of crystalline silicon solar modules by advanced manufacturing, in: Proceedings of the 27th EU PVSEC, Frankfurt, Germany, 2012, pp. 812-815.

[2] D.M. Powell, M.T. Winkler, H.J. Choi, C.B. Simmons, D. Berney Needleman, T. Buonassisi, Crystalline silicon photovoltaics: a cost analysis framework for determining technology pathways to reach baseload electricity costs, Energy Environ. Sci. 5 (2012) 5874-5883.

[3] A. Goodrich, P. Hacke, Q. Wang, B. Sopori, R. Margolis, T.L. James, A waferbased monocrystalline silicon photovoltaics road map: Utilizing known technology improvement opportunities for further reductions in manufacturing costs, Sol. Energy Mater. Sol. Cells 114 (2013) 110-153.

[4] E. Sachs, A. Lorenz, R. Wallace, S. Hudelson, R. Jonczyk, B. Kernan, M. Ismail, D. Harvey, C. Lund, F. van Mierlo, Direct WaferTM - High performance $156 \mathrm{~mm}$ silicon wafers at half the cost of sawn, in: Proceedings of the 28th EU PVSEC Paris, France, 2013, pp. 907-910.

[5] G. Hahn, A. Schönecker, New crystalline silicon ribbon materials for photovoltaics, J. Phys.: Condens. Matter 16 (2004) R1615-R1648.

[6] H. Lange, I.A. Schwirtlich, Ribbon Growth on Substrate (RGS) - A new approach to high speed growth of silicon ribbons for photovoltaics, J. Cryst. Growth 104 (1990) 108-112.

[7] G. Hahn, D. Sontag, C. Haessler, Current collecting channels in RGS silicon solar cells - are they useful? Sol. Energy Mater. Sol. Cells 72 (2002) 453-464.

[8] A. Schönecker, L.J. Geerligs, A. Müller, Casting technologies for solar silicon wafers: block casting and ribbon-growth-on substrate, Solid State Phenom. 95-96 (2004) 149-158.
[9] G. Hahn, S. Seren, M. Kaes, A. Schönecker, J.P. Kalejs, C. Dubé, A. Grenko, C. Belouet, Review on ribbon silicon techniques for cost reduction in PV, in: Proceedings of the 4th WC-PEC, Waikoloa, USA, 2006, pp. 972-975.

[10] B. Heilbronn, F. De Moro, E. Jolivet, E. Tupin, B. Chau, R. Varrot, B. Drevet, S. Bailly, D. Rey, H. Lignier, Y. Xi, T. Riberi-Béridot, N. Mangelinck-Noel, G. Reinhart, G. Regula, Fast growth of thin multi-crystalline silicon ribbons by the RST method, Cryst. Res. Technol. 50 (2015) 101-114.

[11] U. Hess, Investigations of RGS Silicon Solar Cells, Dissertation, University of Konstanz, 2013

[12] P.-Y. Pichon, A. Schönecker, D. Herlach, S. Würzner, SiC filament nucleation and growth in the Ribbon Growth on Substrate (RGS) silicon wafer process, in: Proceedings of the 7th International Workshop on Crystalline Silicon for Solar Cells, Fukuoka, Japan, 2013.

[13] U. Hess, P.Y. Pichon, S. Seren, A. Schönecker, G. Hahn, Crystal defects and thei impact on ribbon growth on substrate (RGS) silicon solar cells, Sol. Energy Mater. Sol. Cells 117 (2013) 471-475.

[14] M.H. AlRifai, O. Breitenstein, JP. Rakotoniaina, M. Werner A. Kaminski, N. LeQuang, Investigation of material-induced-shunts in block-cast multicrystalline silicon solar cells caused by SiC precipitate filaments, in: Proceedings of the 19th EU PVSEC, Paris, France, 2004, pp. 632-635.

[15] J. Bauer, O. Breitenstein, J.P. Rakotoniaina, Electronic activity of SiC precipitates in multicrystalline solar silicon, Phys, status solidi (a) 204 (2007) 2190-2195.

[16] O. Breitenstein, J. Bauer, J.P. Rakotoniaina, Material-induced shunts in multicrystalline silicon solar cells, Semiconductors 41 (2007) 440-443.

[17] S. Seren, M. Kaes, G. Hahn, A. Gutjahr, A.R. Burgers, A. Schönecker, Efficiency potential of RGS silicon from current R\&D production, in: Proceedings of the 22nd EU PVSEC, Milan, Italy, 2007, pp. 854-858.

[18] P.-Y. Pichon, Solidification kinetics versus wetting kinetics relevant to Ribbon, Growth on Substrate (RGS) casting of Silicon, Dissertation, Ruhr-University of Bochum, 2015.

[19] R.A. Sinton, A. Cuevas, M. Stuckings, Quasi-steady photoconductance, a new method for solar cell material and device characterization, in: Proceedings of the 25th IEEE PVSC, Washington, USA, 1996, pp. 457-460.

[20] A.-K. Søiland, Silicon for Solar Cells (Dissertation), Norwegian University of Sience and Technology, 2005.

[21] J. Junge, J. Ebser, S. Graf, B. Terheiden, S. Seren, G. Hahn, M. Käs, Evaluating the efficiency limits of low cost mc Si materials using advanced solar cell processes, in: Proceedings of the 25th EU PVSEC, Valencia, Spain, 2010, pp. 1722 1726.

22] E. Schneiderlöchner, R. Preu, R. Lüdemann, S. Glunz, Laser-fired rear contacts for crystalline silicon solar cells, Prog. Photovoltaics: Res. Appl. 10 (2002) 29-34.

[23] O. Breitenstein, Understanding the current-voltage characteristics of industrial crystalline silicon solar cells by considering inhomogeneous current distributions, Opto-Electron. Rev. 21 (3) (2013) 259-282.

24] P.A. Basore, Extended spectral analysis of internal quantum efficiency, in: Proceedings of the 23rd IEEE PVSC, Louisville, USA, 1993, pp. 147-152.

[25] O. Breitenstein, Nondestructive local analysis of current-voltage characteristics of solar cells by lock-in thermography, Sol. Energy Mater. Sol. Cells 95 (2011) 2933-2936.

[26] O. Breitenstein, Local efficiency analysis of solar cells based on lock-in thermography, Sol. Energy Mater. Sol. Cells 107 (2012) 381-389.

[27] O. Breitenstein, C. Shen, H. Kampwerth, M.A. Green, Comparison of DLIT- and PL-based local solar cell efficiency analysis, Energy Procedia 38 (2013) 2-12.

[28] S. Rißland, O. Breitenstein, High resolution saturation current density imaging at grain boundaries by Lock-in thermography, Sol. Energy Mater. Sol. Cells 104 (2012) 121-124.

[29] O. Breitenstein, J.P. Rakotoniaina, M.H. Al Rifai, M. Werner, Shunt types in crystalline silicon solar cells, Prog. Photovoltaics: Res. Appl. 12 (2004) 529-538.

[30] O. Breitenstein, J.P. Rakotoniaina, J. Schmidt, Comparison of shunt imaging by crystal sheets and Lock-in thermography, in: Proceedings of the 12th Workshop on Crystalline Solar Cell Materials and Processes, Breckenridge, USA 2002, pp. 244-247.

[31] U. Hess, S. Joos, S. Seren, G. Hahn, P.-Y. Pichon, A. Schönecker, T. Weber Infrared microscopy investigation of the crystal structure of ribbon growth on substrate (RGS) solar cells, in: Proceedings of the 24th EU PVSEC, Hamburg, Germany, 2009, pp. 2138-2143.

[32] G.W. Lorimer, Précipitation dans les joints de grains. Precipitation at grain boundaries, Le. J. De. Phys. Colloq. 36 (C4) (1975), C4-233-C4-244.

[33] A.L. Greer, Triple lines in nucleation, Scr. Mater. 62 (2010) 899-903.

[34] T. Duffar, A. Nadri, On the twinning occurrence in bulk semiconductor crystal growth, Scr. Mater. 62 (2010) 955-960.

[35] FEI VisualizationSciences Group (vsg). Avizo Fire 8.0.1. 〈http://www.vsg3d. com/avizo/fire . 